\title{
A Game Theory Based on Monte Carlo Analysis for Optimizing Evacuation Routing in Complex Scenes
}

\author{
Wenhui Li, Jinlong Zhu, Huiying Li, Qiong Wu, and Liang Zhang \\ College of Computer Science and Technology, Jilin University, Changchun 130012, China \\ Correspondence should be addressed to Huiying Li; lihuiying@jlu.edu.cn
}

Received 30 December 2014; Revised 24 April 2015; Accepted 12 May 2015

Academic Editor: Alessandro Salvini

Copyright ( 2015 Wenhui Li et al. This is an open access article distributed under the Creative Commons Attribution License, which permits unrestricted use, distribution, and reproduction in any medium, provided the original work is properly cited.

\begin{abstract}
With more complex structures and denser populations, congestion is a crucial factor in estimating evacuation clearance time. This paper presents a novel evacuation model that implements a game theory combining the greatest entropy optimization criterion with stochastic Monte Carlo methods to optimize the congestion problem and other features of emergency evacuation planning. We introduce the greatest entropy criterion for convergence to Nash equilibrium in the $n$-person noncooperative game. The process of managing the conflict problem is divided into two steps. In the first step, we utilize Monte Carlo methods to evaluate the risk degree of each route. In the second step, we propose an improved method based on game theory, which obtains an optimal solution to guide the evacuation of all agents from the building.
\end{abstract}

\section{Introduction}

Emergency evacuation plans are developed to ensure the safest and most efficient evacuation time of all expected residents of a structure or region [1-4]. With the increasing complexity of building and frequency of disasters, the evacuation routing optimization problem has become very popular in the area of emergency planning. In particular, evacuee congestion-related evacuation modeling has drawn attention because evacuee congestion has a significant impact on emergency evacuation planning. Ha and Lykotrafitis [5] considered motivational force, psychological repulsive tendency, compression, viscous damping/personal force, and sliding friction in the simulation of specific emergency evacuations. They govern particles' motion by the social-force model to investigate the effect of crowd evacuation. Chooramun et al. [6] developed an evacuation model utilizing hybrid space discretization, which uses a mixture of three basic techniques for space discretization, namely, coarse networks, fine networks, and continuous networks. Manley and Kim [7] took an agent-based approach to estimate formation of bottlenecks during urgent evacuation. Furthermore, many studies have been conducted to interpret the multiple-exit selection problem in a game-theoretic framework. The work of Zheng et al. [8-10] studied evacuees' cooperative and competitive behaviors by using a close analogy to the chicken-type game. Tanimoto et al. [11] proposed a deductive approach to analyze the bottleneck problems of pedestrian evacuation by using a close analogy to the saint and temptation reciprocity game. Shi and Wang [12] proposed a microscopic framework to research the complicated interactions among the competing pedestrians based on the modified lattice gas model by using snowdrift game theory. Lo et al. [13] proposed a dynamic exit selection model by calculating a mixed-strategy Nash equilibrium of a zero-sum game. Li et al. [14] proposed a Bayesian game to research how pedestrians select exits for evacuation optimization. They proposed a QRA model only considering one factor with Monte Carlo methods. They regarded the individual as a participant and prepared a local framework to research the evacuation problem; however, this method omits global evacuation factors. During the past decade, game theory has been developed in the areas of competitive behaviors and the rationality problem of evacuees [15-18]. However, the degree of congestion of the emergency exit, the average congestion degree of the evacuation route, the congestion degree of diverted traffic, 
the route evacuation degree, the maximum flow rate of exit, and the fire origin location are six crucial factors that affect evacuation clearance time and the process of evacuation. In most evacuation models, modeling the dynamic wayfinding of people with respect to these congestion situations and the actions of other evacuees simultaneously is rare. Therefore, the present study is an attempt to present a new evacuation routing optimization from the perspective of these six factors.

In this paper, we present a multiple-exit evacuation model (MEEM) based on a game with quantitative risk assessment (QRA) to make an optimal egress route plan, taking into account these six factors. MEEM uses information about the congestion and evacuees to evaluate the risks and compute the Nash equilibrium of the evacuees using the payment function of the game. To demonstrate our method, the crowd simulations treat all evacuees as discrete individuals using agent-based modeling (ABM) [5]. ABM considers each individual as a particle whose motion is governed by Newton's equations, assuming that we know the agents' distribution and locations. We measured experimental data from the first floor of the Computer Building in Jilin University.

This paper addresses two issues. The first is how to estimate the degree of risk of a route. The second is how to determine the optimal evacuation routes for evacuees. This paper investigates the optimal evacuation plan using a gametheoretical model to estimate the degree of risk of a route. The evacuation clearance time and crowd density can be improved using this technique, which also takes full advantage of multiple exits to obtain the safest evacuation route.

The remainder of this paper is organized as follows. Section 2.1 introduces the QRA model based on Monte Carlo methods. Section 2.2 presents the route optimization based on game theory and introduces the related work about evacuation planning. Section 3 presents the optimization results. Section 4 concludes this paper and looks into the future of this research.

\section{The Methodology}

2.1. Risk Assessment. Quantitative risk assessment models based on the Monte Carlo algorithm were designed to estimate the risk indices for the routes, which are used in quantitative analysis and decision-making. They involve a class of computational algorithms that rely on repeated random sampling to calculate their results. Some researchers use Monte Carlo method for simulating pedestrian evacuation $[2,4,19-21]$. They are often used to model phenomena with significant uncertainties in inputs.

Monte Carlo simulation furnishes the decision-maker with a range of possible outcomes and associated probabilities. They will occur for any choice of action. It shows the extreme possibilities of "going for broke" as well as the most conservative decision along with all conceivable consequences in between.

This simulation method does not always require truthful data to be computed, such as risk assessment. We use deterministic and pseudorandom sequences, making it easy to test and rerun simulations. The only quality usually necessary to make satisfactory simulations is the pseudorandom sequence to appear "random enough" in a certain sense. The model follows a particular pattern:

(1) Define a domain of possible inputs for the risk assessment.

(2) Randomly generate inputs from a probability distribution sampled over the domain.

(3) Perform a deterministic estimate on the inputs according to distribution characteristics of the random variable.

(4) Obtain the risk assessment value.

The quantitative risk assessment principles estimate the risk indices of the escape route by combining the various risk weights of enclosures. We select sensitive factors as stochastic variables in the Monte Carlo analysis. The factors consist of five principal events: the fire origin location, the congestion degree $c_{\text {exit }}$ of the emergency exit, the average congestion degree $c_{\text {route }}$ of the evacuation route, the congestion degree $c_{\text {intersection }}$ of diverted traffic, and the route evacuation degree $t_{\text {evacuation }}$.

Here, $t_{\text {evacuation }}=d_{\text {exit }} / s_{\text {room }}: d_{\text {exit }}$ is the distance between the exit and the location and $s_{\text {room }}$ is the average speed of the pedestrians in an enclosure. The congestion degree is the $D$ value between the congestion evacuation time and the ideal evacuation time. The building construction regulates the minimum requirement of outflow rate per unit exit width and unit time, called the outflow coefficient rate evacuate $_{\text {. The ideal }}$ evacuation time is the quotient of the number of pedestrians

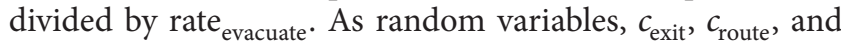
$t_{\text {evacuation }}$ obey lognormal distributions in the QRA model, and $c_{\text {intersection }}$ obeys a triangular distribution.

The triangular distribution is defined using the most optimistic value $\mathrm{OP}$, the maximum possible value $\mathrm{MP}$, and the most pessimistic value $\mathrm{PP}$, where $0<\mathrm{OP}<\mathrm{MP}<$ $\mathrm{PP}$. The values around the maximum possible value are more likely to occur. The variables described by a triangular distribution include the congestion per unit of time. The triangular distribution data of congestion degree of diverted traffic are shown in Table 1.

The QRA model calculates OP, MP, and PP for the duration time of the key congestion crossroads. $Y=\left(X_{1}+\right.$ $\left.X_{2}+\cdots+X_{n}\right) / n, c v$ (coefficient of dispersion) $=\sigma / Y$, and cv $<10 \%$.

The lognormal distribution is defined using values that are positively skewed, not symmetric like a normal distribution. It is used to represent the values that do not go below zero but have unlimited positive potential. For a lognormal distribution $X$, the parameters denoted by $\mu$ and $\sigma$ are, respectively, the mean and the standard deviation of the variable's natural logarithm, $X=e^{\mu+\sigma Z}$, and $Z$ is a standard normal variable. Norm $=$ NORMSINV $($ LAP), NORMSINV is the inverse function of the standard normal distribution function, and $\sigma_{i}=-R_{i} /$ Norm. See Table 2 for the congestion degree of the emergency exit.

According to the distribution function, we parse the inverse function of the random variable. Then, we calculate 
TABLE 1: Triangular distribution function and inverse function.

\begin{tabular}{lcccccc}
\hline Order & OP & MP & PP & $\left(A-Y_{a}\right)^{2} / n$ & $\left(B-Y_{b}\right)^{2} / n$ & $\left(C-Y_{c}\right)^{2} / n$ \\
\hline 1 & 25 & 50 & 99 & 0.036 & 0.036 & 0.016 \\
2 & 25 & 50 & 100 & 0.036 & 0.036 & 0.036 \\
3 & 26 & 51 & 98 & 0.016 & 0.016 & 0.196 \\
4 & 27 & 52 & 102 & 0.196 & 0.196 & 0.676 \\
5 & 25 & 50 & 100 & 0.036 & 0.036 & 0.036 \\
6 & 26 & 51 & 99 & 0.016 & 0.016 & 0.016 \\
7 & 25 & 50 & 100 & 0.036 & 0.036 & 0.036 \\
8 & 27 & 52 & 97 & 0.196 & 0.196 & 0.576 \\
9 & 25 & 50 & 99 & 0.036 & 0.036 & 0.016 \\
10 & 25 & 50 & 100 & 0.036 & 0.036 & 0.036 \\
$Y$ & 25.6 & 50.6 & 99.4 & 0.64 & 0.64 & 1.64 \\
$\sigma$ & & & & 0.8 & 0.8 & 1.280625 \\
$c v$ & $3.13 \%$ & $1.58 \%$ & $1.29 \%$ & & & \\
\hline
\end{tabular}

TABLE 2: Lognormal distribution function and inverse function.

\begin{tabular}{lcccccccc}
\hline Order & Min & Max & CP & $R$ & LAP & Norm & $\mu$ & $\sigma_{i}$ \\
\hline 1 & 30 & 54 & 0.9 & 12 & 0.05 & -1.645 & 42 & 7.29548 \\
2 & 31 & 55 & 0.85 & 12 & 0.075 & -1.440 & 43 & 8.33604 \\
3 & 31 & 55 & 0.9 & 12 & 0.05 & -1.645 & 43 & 7.29548 \\
4 & 32 & 56 & 0.9 & 12 & 0.05 & -1.645 & 44 & 7.29548 \\
5 & 31 & 55 & 0.85 & 12 & 0.075 & -1.440 & 43 & 8.33604 \\
6 & 30 & 54 & 0.85 & 12 & 0.075 & -1.440 & 42 & 8.33604 \\
7 & 32 & 56 & 0.95 & 12 & 0.025 & -1.960 & 44 & 6.12256 \\
8 & 30 & 54 & 0.9 & 12 & 0.05 & -1.645 & 42 & 7.29548 \\
9 & 31 & 55 & 0.9 & 12 & 0.05 & -1.645 & 43 & 7.29548 \\
10 & 30 & 54 & 0.85 & 12 & 0.075 & -1.440 & 42 & 8.33604 \\
$Y$ & & & & & & & 42.8 & 7.59441 \\
$\sigma$ & & & & & & & 0.748 & 0.69377 \\
$c v$ & & & & & & & $1.75 \%$ & $9.14 \%$ \\
\hline
\end{tabular}

Min: the congestion minimum value, Max: the congestion maximum value, and CP: coverage probability.

the results iteratively, each time drawing a different set of random deviates from the probability functions. Five hundred Monte Carlo simulation iterations are used. Finally, we calculate the mean value of each event as the tabular statement of assessment criteria (TSAC).

In this section, we propose a risk index (RI) to quantify the magnitude of risk for emergency exits based on TSAC. As a general case, we consider an example of an enclosure that has $N$ exits leading to the outside of the building. Let $\mathrm{RI}=p_{i} / \sum p_{j}, i \in N$ and $j \in N, p_{i}$ representing that the $i$ th exit has imposed capacity restrictions. We thus have

$$
p_{i}(x)=\min \left(f_{i}(x), c_{i}\right) .
$$

Here, $x$ is the number of pedestrians in the enclosure and $c_{i}$ is the threshold of the pedestrian in the $i$ th exit making sure that every exit congestion time is less than the congestion time threshold (20 seconds). We calculate each exit that can accommodate a maximum number of evacuated people $f(x)$, concretely as

$$
f(x)_{i}= \begin{cases}t_{\text {safe }} * s_{\text {room }} & \left(t_{\text {safe }}>0, t_{\text {safe }}<t_{\text {evacuation }}\right), \\ x & \left(t_{\text {safe }}>0, t_{\text {safe }}>t_{\text {evacuation }}\right), \\ 0 & \left(t_{\text {safe }}<0\right),\end{cases}
$$

where $t_{\text {safe }}=t_{\text {harm }}-Q_{\text {risk }}$, and $Q_{\text {risk }}$ is defined as

$$
Q_{\text {risk }}=c_{\text {intersection }}+t_{\text {evacuation }}+c_{\text {exit }}+c_{\text {path }} \text {. }
$$

Here, $t_{\text {harm }}$ is the available safe egress time:

$$
t_{\text {harm }}=\frac{t_{c} * d}{f_{r}},
$$

where $t_{c}$ is the duration time of the combustion source, $d$ is the distance between the fire location and the exit, and $f_{r}$ is the risk radius of the combustion source.

$\mathrm{RI}$ is a weighting factor of payoff function in MEEM, allocating the distribution of evacuees in each route using game theory.

2.2. Route Optimization Based on Game Theory. A game consists of a set of participants, a set of strategies available to those participants, and a specification of payoffs for each combination of strategies [22].

In this paper, we present MEEM by using a close analogy to the $n$-person noncooperative game. MEEM provides a macroscopic perspective to understand the conflicts during emergency evacuation as well as the rationality of evacuees. We employ a density-detection method to judge a congestion problem. The density-detection method employed assesses the crowd density during emergency evacuations in cases where the rectangular enclosures are discrete homogeneous building enclosures. If the crowd density of rectangular enclosures is above the density threshold, we regard all escape exits as the participants of the game. We define any combination of distributed evacuations of each participant as game strategies. The maximum flow rate of each exit is the number of agents walking through an exit per unit time. The maximum flow rate (MFR) can influence the evacuation time. For example, when a number of evacuees who decide to egress through an exit come to the MFR, the evacuees must queue at the exit. The corridor has a maximum flow rate, like the exit. In order to introduce the MFR, we set the ratio $\sigma$ of the MFR of the exit to the MFR of the corridor as a weighting factor in the payoff function.

The enclosure calculates evacuation planning using game theory with Monte Carlo risk assessment mechanisms. The evacuation planning redistributes agents to other exits in the queue, which reduces queue sizes and the escape time.

The conflict problem uses MEEM that has two significantly different processes in two different phase states. The first is a preoptimization state, in which the Dijkstra algorithm obtains the shortest route of each enclosure (e.g., a room) to an exit at the beginning of the evacuation. The second is a conflict optimizing state, in which crowd densities 
over the density threshold $d_{\text {threshold }}$ trigger reselection of other exits to reduce evacuation clearance time.

The process of an agent's evacuation is mainly influenced by other agents and the environment. Each agent will always choose the maximum utility (minimum cost) route with respect to the states of the exits so as to minimize the escape time. In the preoptimization state, each exit estimates the crowd density according to the expected travel time of all enclosures and the outflow coefficient. If the crowd density of an exit will exceed the density threshold, we treat an enclosure as a whole entity and assign all the evacuees of the enclosure to the exit.

In the conflict optimizing state, we treat all the evacuees of crowding as a whole entity and reselect any exit, when the crowding is formed at the vicinity of an exit in the evacuation process. The distance to the reselected exit is less than the scope threshold between the reselected exit and the enclosure.

As a general case, we consider the MEEM in that $P$ pedestrians are anxious to evacuate from an enclosure. The enclosure has $N$ exits leading to the outside of the building. The initial positions of the evacuees are distributed randomly at the start of evacuation. The game is defined as $G=$ $\{R, S, A, U\}$, where

(1) participator is the $i$ th escape exit defined as $R_{i}, i \in N$, $N=\{1,2, \ldots, n\}$;

(2) strategy space is a strategy of $R_{i}$ which is $S_{i}, S_{i} \in$ $\left[0, G_{i}\right], i \in N$ and we assume that the largest number of evacuees is $G_{i}$ from $R_{i}$ and $G_{i}$ is equal to $p_{i}(x)$ in QRA method;

(3) action is $B_{i}$ which is the set of actions for enclosure $i$; let $B=\{$ select, select none $\}, i \in N$;

(4) preference is the priority level of the game such that more dangerous routes obtain higher priority;

(5) $U\left(x_{1}, \ldots, x_{n}\right)$ is the payoff function of the game, where $p_{i}-q_{i} * x_{i}$ represents the payoff value of $R_{i}$; more formally, let

$$
U\left(x_{1}, \ldots, x_{n}\right)= \begin{cases}\left(p_{1}-q_{1} x_{1}, \ldots, p_{n}-q_{n} x_{n}\right), & 0 \leq x_{1} \leq G_{1}, \ldots, 0 \leq x_{n} \leq G_{n}, 0 \leq x_{1}+\cdots+x_{n} \leq P \\ (0, \ldots, 0), & \text { other, }\end{cases}
$$

where $p_{i}=\delta_{i} * \mathrm{RI}_{i} * P, p_{i}=\delta_{i}$, and $p_{i}-q_{i} x_{i}$ is satisfied by

$$
\sigma *(\mathrm{RI} * P-x)
$$

The product of RI times $P$ represents the maximal number of evacuees in the $i$ th exit. Based on the QRA method, we can estimate the number of occupants evacuated at the $i$ th exit by the product of RI and $P$. $x$ represents the number of evacuees who set the $i$ th exit as the destination. When $x$ increases, $\mathrm{RI} * P-x$ decreases and so does the revenue. In addition, we consider the parameter $\sigma$ as the outflow coefficient to affect the evacuation planning. When $\sigma<1$, it illustrates that occupants who walk through the corridor to the exit are more numerous than those who escape through the exit. With the decrease of $\sigma$, the capacity of evacuation becomes smaller, and it is more than 0 . When $\sigma$ is equal to 1 , it indicates that the corridor and the exit have an outflow coefficient of the same width. Therefore, we take $\left(p_{i}-q_{i} * x_{i}\right)$ to influence the capacity of evacuation at each exit in terms of the evacuation rate.

In game theory, a Nash equilibrium is the optimal combination of strategies. It will force the strategies proposed by some participants to be the best reaction to strategies put forward by other participants. We introduce the Greatest Entropy Criterion (GEC) for solving the Nash equilibrium involved in the game theory.

The GEC shows that every player must understand what strategies other players select in order to obtain large amounts of information. Nash equilibria are expected equilibria in $n$-person noncooperative condition games under GEC. The Nash equilibrium is the maximum point of $G$. We set $\left(a_{1}^{*}, \ldots, a_{n}^{*}\right)$ as the expected equilibrium; $a_{j}^{*}$ is the maximum point of the function $G_{j}\left(a_{j}\right)$ with the following formulation:

$$
G_{j}\left(a_{j}\right)=\sum_{i \in M / L_{j}} u_{i j}\left(a_{j}\right) \mu\left(F_{i j}\left(a_{j}\right)\right), \quad j=1,2, \ldots, n,
$$

where $L_{j}=\left\{i \in M \mid \mu_{i j}\left(a_{j}\right)=0, \forall a_{j} \in D_{i j}\right\} . M$ is the number of measurable sets on the measurable space, $M \in$ $\{1,2, \ldots, m\}$, and $D_{i j}=\left\{a_{j} \in A_{j} \mid F_{i j}\left(a_{j}\right) \neq \varnothing\right\}$. When each player chooses an action, $A_{j}$ is a payoff function $U\left(x_{1}, \ldots, x_{n}\right)$ for player $j$. In this study, we solve the maximum point with its derivative as follows:

$$
\begin{aligned}
& \frac{d\left[u_{1 j}\left(x_{j}\right) \mu\left(F_{1 j}\left(x_{j}\right)\right)\right]}{d x_{j}} \\
& \quad=u_{1 j}\left(x_{j}\right) \frac{d \mu\left(F_{1 j}\left(x_{j}\right)\right)}{d x_{j}}+\mu\left(F_{1 j}\left(x_{j}\right)\right) \frac{d u_{1 j}\left(x_{j}\right)}{d x_{j}} .
\end{aligned}
$$

The detailed mathematical description of the derivative of function $G_{j}\left(a_{j}\right)$ is given by

$$
\begin{aligned}
& \mu\left(F_{1 j}\left(x_{j}\right)\right) \\
& \quad=\mu\left\{\left(x_{1} \in\left[0, G_{1}\right], \ldots, x_{n} \in\left[0, G_{n}\right]\right) \mid \sum_{k=1, k \neq j}^{n} x_{k}\right. \\
& \left.\quad \leq P-x_{j}\right\}=\frac{1}{(n-1) !}\left(P-x_{j}\right)^{n-1} .
\end{aligned}
$$




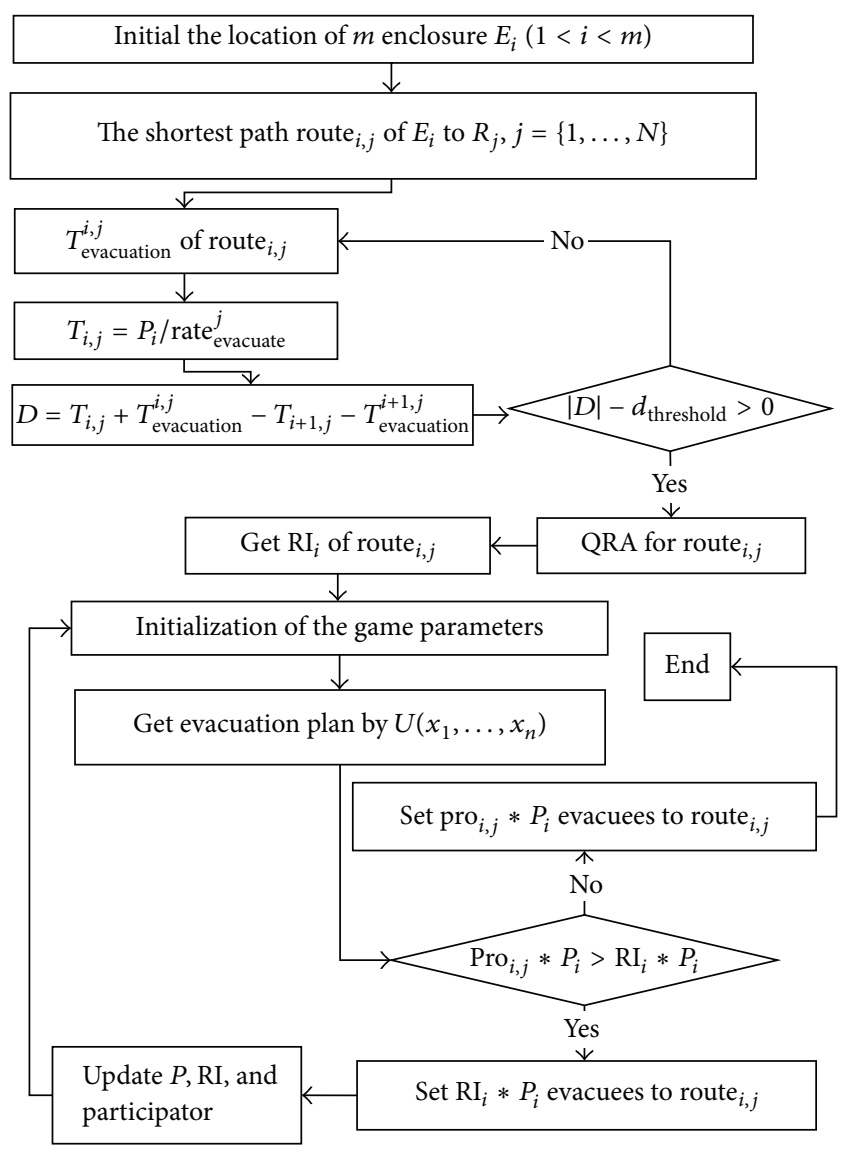

FIgURE 1: Game theory to solve the congestion process.

We see that the variable $G_{j}\left(a_{j}\right)$ becomes

$$
\begin{aligned}
u_{1 j}\left(x_{j}\right) & =p_{j}-q_{j} x_{j}, \\
\frac{d \mu\left(F_{1 j}\left(x_{j}\right)\right)}{d x_{j}} & =-\frac{1}{(n-2) !}\left(P-x_{j}\right)^{n-2}, \\
u_{2 j}\left(x_{j}\right) & =0 .
\end{aligned}
$$

The derivative of the function $G_{P}\left(a_{P}\right)$ is

$$
\begin{aligned}
& \frac{d\left[u_{1 j}\left(x_{j}\right) \mu\left(F_{1 j}\left(x_{j}\right)\right)\right]}{d x_{j}} \\
& \quad=\frac{1}{(n-1) !}\left(P-x_{j}\right)^{n-2}\left[n q_{j} x_{j}+p_{j}-n p_{j}-P q_{j}\right] .
\end{aligned}
$$

We can solve for $x_{j}$. Let pro $_{j}=x_{j} / \Sigma x_{n}$ and obtain the

\begin{tabular}{|c|c|c|c|}
\hline & Agent model [5] & MEEM & Exit selection model [13] \\
\hline \multicolumn{4}{|l|}{$P$} \\
\hline Exit 1 & 160 & 116 & 130 \\
\hline Exit 2 & 0 & 44 & 30 \\
\hline$T$ & 89 & 77 & 84 \\
\hline
\end{tabular}
pedestrian distribution $\operatorname{pro}_{j} * P$ assigning the crowd to $R_{j}$. Figure 1 shows the game theory rules to deal with the conflict. The computation process will be iterated at each time step.

\begin{tabular}{|c|c|c|}
\hline & $\sigma=0.5$ & $\sigma=1$ \\
\hline \multicolumn{3}{|l|}{$P$} \\
\hline Exit 1 & 116 & 152 \\
\hline Exit 2 & 44 & 8 \\
\hline$T$ & 77 & 57 \\
\hline
\end{tabular}

TABLE 3: Exiting pattern for a 4-room/1-corridor situation.

TABLE 4: Exiting pattern for different outflow coefficient ratio situation.

\section{Discussion and Results}

The configurations for the simulation are set as follows:

(1) The room is a square space, $10 \mathrm{~m} \times 15 \mathrm{~m}$. The corridor is a square space, $40 \mathrm{~m} \times 4 \mathrm{~m}$.

(2) There are 40 pedestrians in each room with a total of 160 pedestrians in the entire building.

(3) The fire location is in the middle of a long corridor space. The fire and the corridor are $18 \mathrm{~m}$ apart.

(4) We set the parameters of fire as follows: $f_{r}=2, t_{c}=$ 10 , and $d=20 \sqrt{2}$.

Figure 2 shows four rooms (black squares), a corridor (gray square), four doors (red squares), and two escape exits (purple squares). The average escape time for the agent, MEEM, and exit selection models are 89 minutes, 77 minutes, and 84 minutes, respectively.

Table 3 shows the distribution of evacuees among two exits. We can see that MEEM makes the most of the availability of the two exits. The simulation output with the game theory model provides a more rational result than the agent method by merely considering the travel distance from the exit. Our algorithm is compared to the agent method to reduce the evacuation time and the degree of congestion.

Table 4 shows the simulation output of different outflow coefficient ratios for the first exit in the game. When $\sigma$ is closer to 1 , the evacuation capability is larger. With an increase of the outflow coefficient ratio, the evacuation time becomes smaller but is not more than 1 . The experiment demonstrated that the referenced evacuation coefficient can affect the evacuation planning.

Figure 3 shows that the simulated output of the two methods gives the same evacuation time when $P$ is less than 80. That is because the congestion degree is less than the density threshold, and the optimized evacuation route can be found without using game theory. With the increase of $P$, the time difference of the two methods becomes greater but is not more than 260 . When 260 pedestrians evacuate from 
TABLE 5: Evaluation index table.

\begin{tabular}{lcccccccc}
\hline \multirow{2}{*}{ Number } & \multicolumn{2}{c}{ Export congestion } & \multicolumn{2}{c}{ Path congestion } & \multicolumn{2}{c}{ Intersection congestion } & \multicolumn{2}{c}{ Path time-consuming } \\
& $\mathrm{RN}$ & $c v$ & $\mathrm{RN}$ & $c v$ & $\mathrm{RN}$ & $\mathrm{cv}$ & $\mathrm{RN}$ & $\mathrm{cv}$ \\
\hline 1 & 0.499257 & 42.79 & 0.710921 & 14.45 & 0.285449 & 7.10 & 0.96203 \\
2 & 0.405381 & 40.98 & 0.983167 & 19.38 & 0.662515 & 8.84 & 0.034211 \\
3 & 0.794601 & 49.05 & 0.983125 & 19.38 & 0.294659 & 7.15 & 0.416339 & 98.16 \\
4 & 0.763606 & 48.25 & 0.564434 & 13.21 & 0.929786 & 10.70 & 0.03238 & 25.91 \\
5 & 0.419017 & 41.25 & 0.147974 & 9.41 & 0.260629 & 6.97 & 0.127017 & 26.21 \\
6 & 0.747297 & 47.86 & 0.67011 & 14.09 & 0.89551 & 10.32 & 0.66539 & 98.69 \\
7 & 0.259172 & 37.89 & 0.368653 & 11.64 & 0.89474 & 10.31 & 0.652972 & 98.68 \\
8 & 0.025218 & 27.94 & 0.038118 & 7.12 & 0.41223 & 7.71 & 0.671424 & 98.70 \\
9 & 0.344342 & 39.76 & 0.445005 & 12.26 & 0.30068 & 7.18 & 0.487949 & 98.52 \\
$\vdots$ & $\vdots$ & $\vdots$ & $\vdots$ & $\vdots$ & $\vdots$ & $\vdots$ & $\vdots$ \\
500 & 0.193843 & 26.36 & 0.193843 & 26.36 & 0.193843 & 26.36 & 0.193843 \\
Mean & 0.50896 & 43.02019 & 0.468052 & 12.38077 & 0.476657 & 7.968394 & 0.50492 & 73.96422 \\
\hline
\end{tabular}

$\mathrm{RN}$ : random number, $\mathrm{RN} \in[0,1]$, and $c v$ : corresponding value.

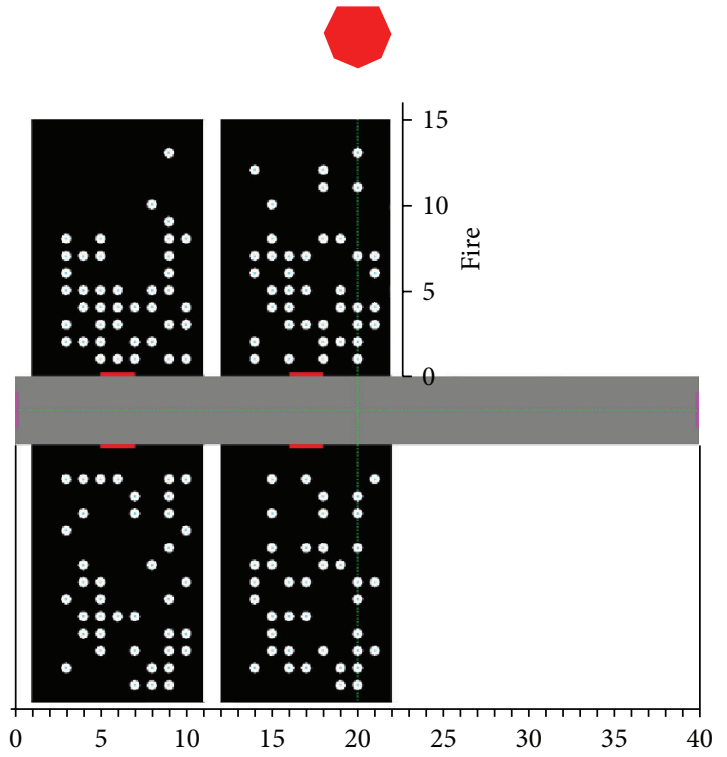

FIGURE 2: Study of the evacuation of the building with four rooms.

the entire building, the agent using game theory is able to push the optimization ability to its limits.

To test our algorithm, we ran it on the first floor of the Computer Building in Jilin University as shown in Figure 4. Figure 4 shows the layout plan of the first floor. The building information, labeled on the diagram, is as follows.

Here, $A(B)$ represents an enclosure $A$ which will accommodate $B$ pedestrians in the circle. Exit $C / D$ describes exit information as a serial number divided by the outflow coefficient of the exit. All occupants will escape from the enclosure and then pass through the exit to reach the place of safety.

The gray rectangles are the corridors of the building. We mark the outflow coefficient of the corridor on its inside.

For example, Exit 1 is an escape hatch in the shortest route of enclosure 15. When the crowd is formed at the vicinity of

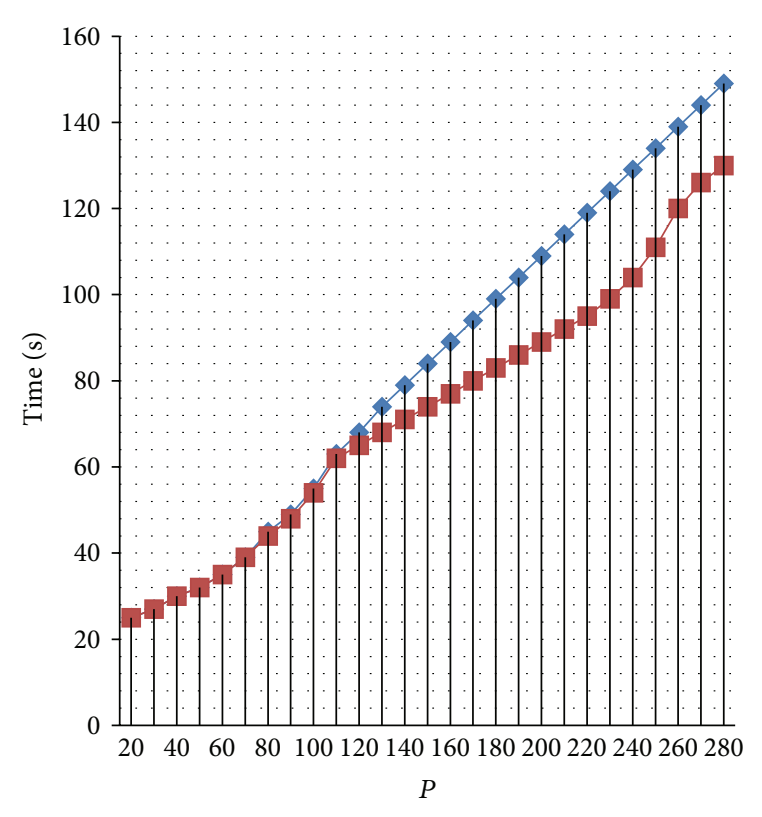

$\rightarrow$ Agent

$\rightarrow$ GQMESM

Figure 3: Average escape time by agent and MEEM.

Exit 1, enclosure 15 will seek an alternative exit. The optional exits include Exit 1, Exit 2, Exit 3, and Exit 4. Figure 6 shows the evacuation assessment of each exit. The red curve represents the number of congestion agents at the exit, and the blue curve represents the number of agents at the exit.

The QRA method calculated the RI of each route according to the threshold of the pedestrian. It generated the tabular statement of assessment criteria for escape routes as shown in Tables 5 and 7.

The simulation output provides a more rational result than merely considering the travel distance to the exit. The total number of evacuees is 806. Table 6 shows 
TABLE 6: Evacuation allocation table.

\begin{tabular}{|c|c|c|c|}
\hline & Section & Headcount & Exit \\
\hline Pretreatment & $\begin{array}{l}1(5), 2(5), 3(5), 4(5), 5(5), 6(5), 7(5), 8(5), 9(5), 10(6), 11(7), 12(6), 13(8), 14(100), 15(100), 31(2) \\
\quad 32(100), 33(100), 34(35), 35(35), 36(35), 37(6), 38(5), 39(5), 40(5), 41(5), 42(5), 43(5)\end{array}$ & 615 & \multirow[t]{2}{*}{1} \\
\hline Postoptimality & $1(5), 10(6), 31(2), 32(100), 33(79), 34(35), 35(35), 37(6), 38(5), 40(5), 41(5), 42(5), 43(5)$ & 293 & \\
\hline Pretreatment & 16(10), 17(10), 18(10), 19(10), 20(10), 21(60), 27(10), 28(8), 29(6), 30(7) & 141 & \multirow[b]{2}{*}{2} \\
\hline Postoptimality & $\begin{array}{c}2(3), 3(3), 4(3), 5(3), 6(3), 7(3), 8(3), 9(3), 11(5), 12(2), 13(6), 14(60), 15(66), 16(10), 17(10), 18(10) \\
19(10), 20(10), 21(60), 27(10), 28(8), 29(6), 30(7), 33(21), 36(21), 39(3)\end{array}$ & 349 & \\
\hline Pretreatment & $22(10), 23(10)$ & 20 & \multirow[b]{2}{*}{3} \\
\hline Postoptimality & $\begin{array}{c}2(1), 3(1), 4(1), 5(1), 6(1), 7(1), 8(1), 9(1), 11(1), 12(2), 13(1), 14(20), 15(17), 22(10), 23(10), 36(7), \\
39(1)\end{array}$ & 77 & \\
\hline Pretreatment & $24(10), 25(10), 26(10)$ & 30 & \multirow[b]{2}{*}{4} \\
\hline Postoptimality & $\begin{array}{c}2(1), 3(1), 4(1), 5(1), 6(1), 7(1), 8(1), 9(1), 11(1), 12(2), 13(1), 14(20), 15(17), 24(10), 25(10), 26(10), \\
36(7), 39(1)\end{array}$ & 87 & \\
\hline
\end{tabular}

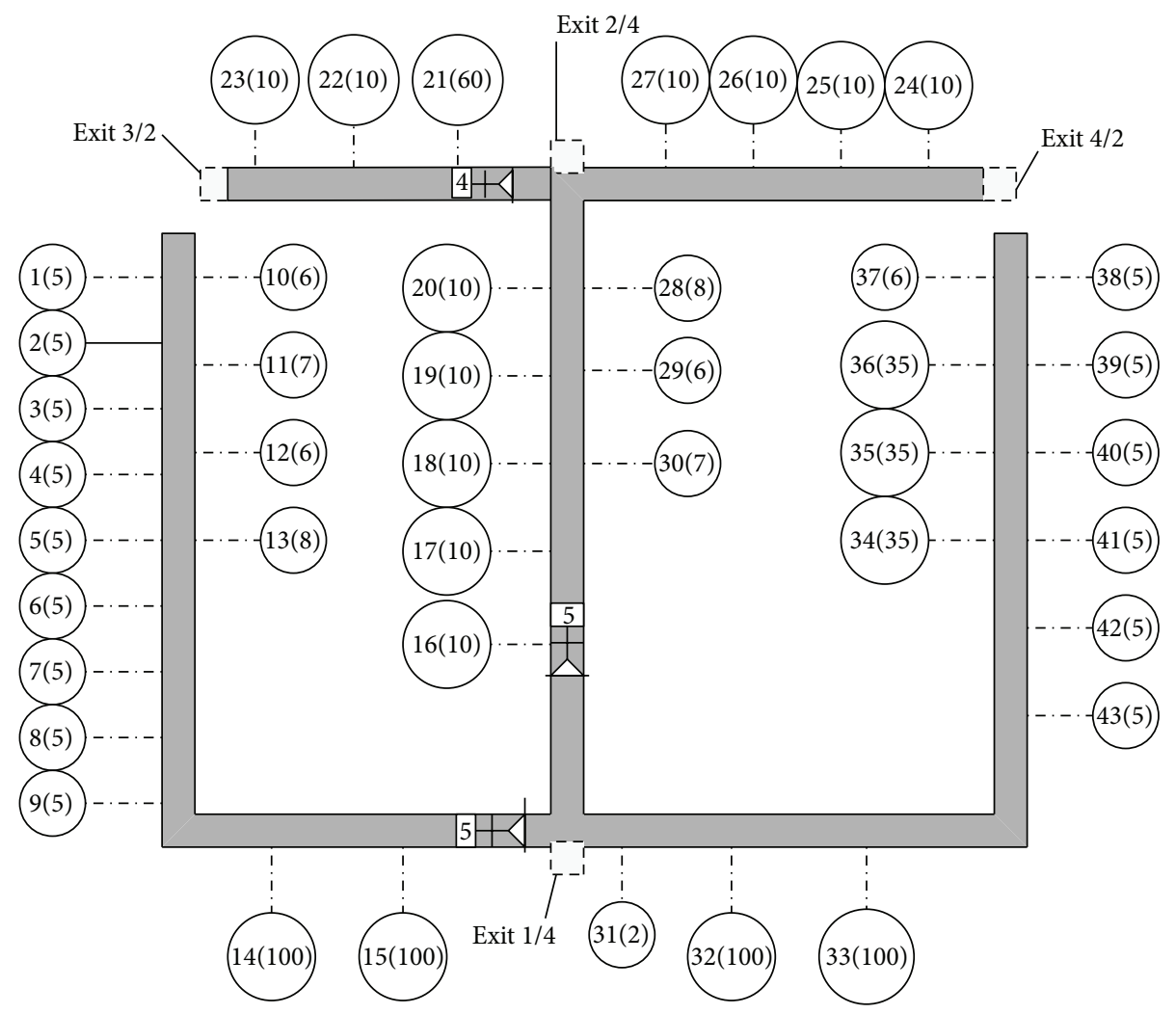

FIgURE 4: Layout plan.

the distribution of the crowd evacuation. The digits are the serial number of the enclosure in front of the brackets of the section column, and the quantitative distribution of the pedestrians is in the brackets. The Headcount column is the total number of pedestrians.

We analyzed the data merely considering the travel distance from the exit and game theory to optimize the export evacuation. The upper half of Figure 7 is the result of the merely agent-based optimization evacuation. The lower half is the details of the evacuation plan. Subsequently, congestion data and evacuation data are described as ways for analyzing the evacuation in Figure 5. Figure 8 is the optimized evacuation diagram. Comparing the two optimized kinds of data shows that the merely agent-based optimization evacuation cost is $161 \mathrm{~s}$ and the maximum congestion number is 332 pedestrians, while the optimized game evacuation cost is 121 s. Meanwhile, we can see from Figure 8 that the evacuation takes full advantage of the four exits and the congestion number is not more than 37 .

Finally, comparing a simulation output for the snowdrift game theory and the exit selection model, we note that those two methods improve the evacuation planning better than 


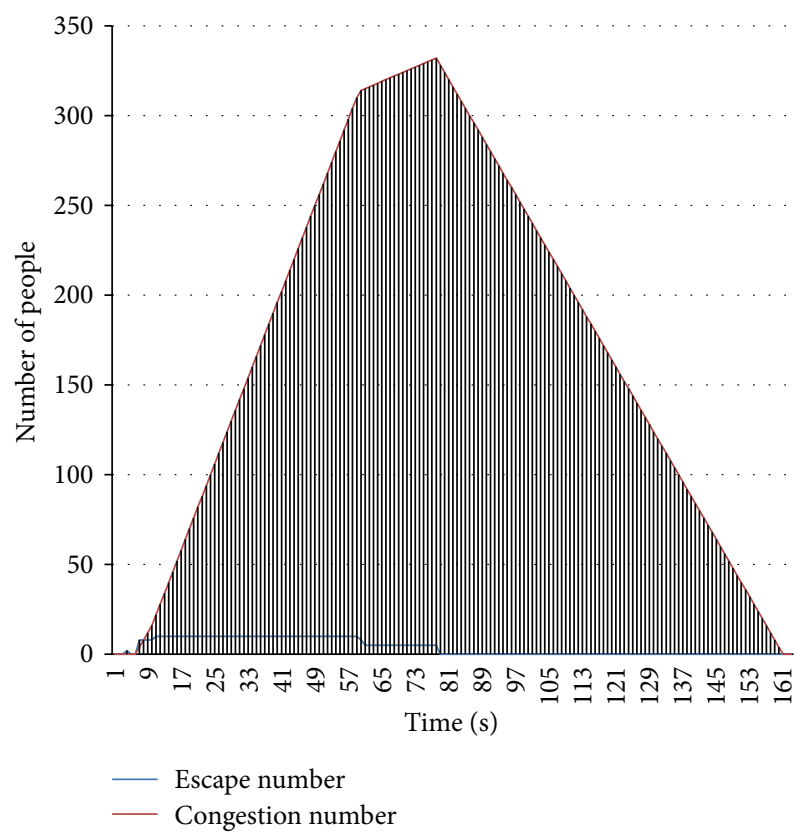

(a) Exit 1

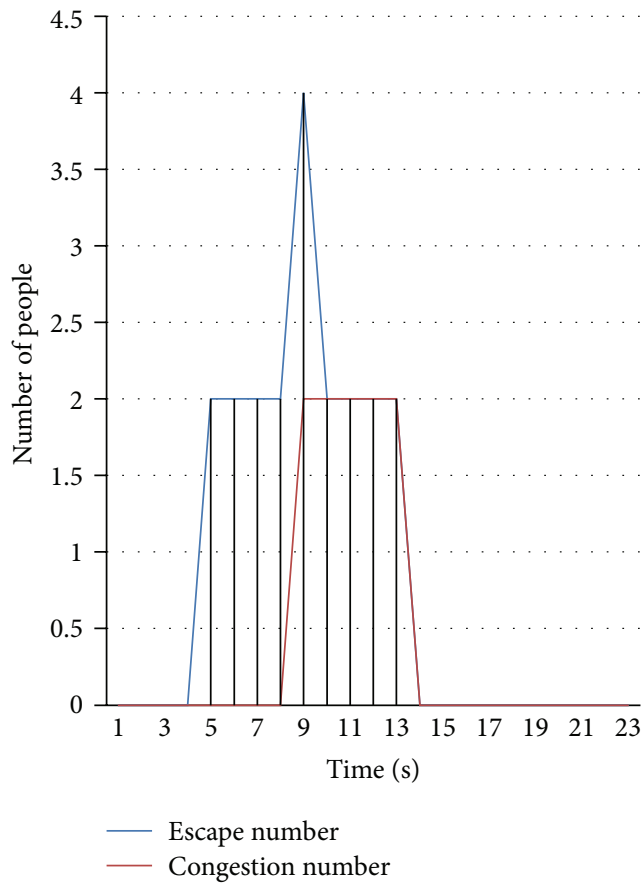

(c) Exit 3

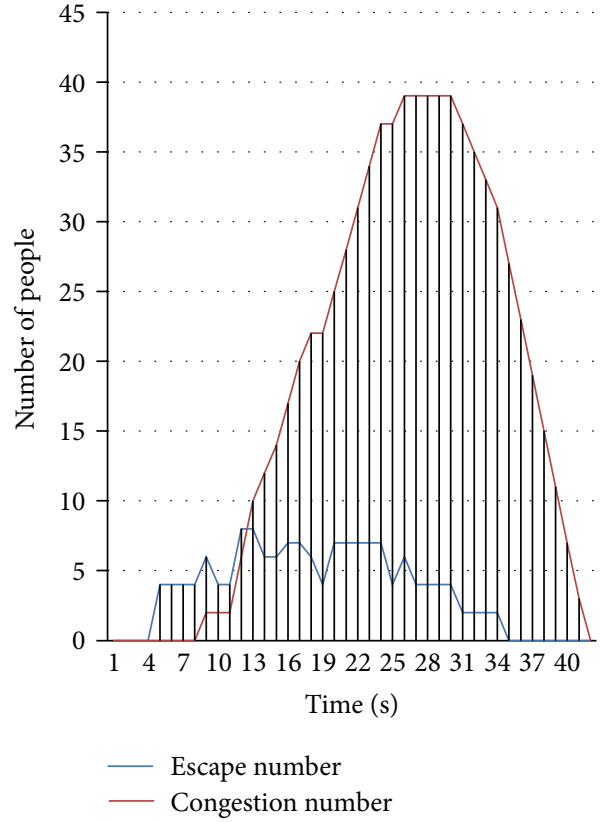

(b) Exit 2

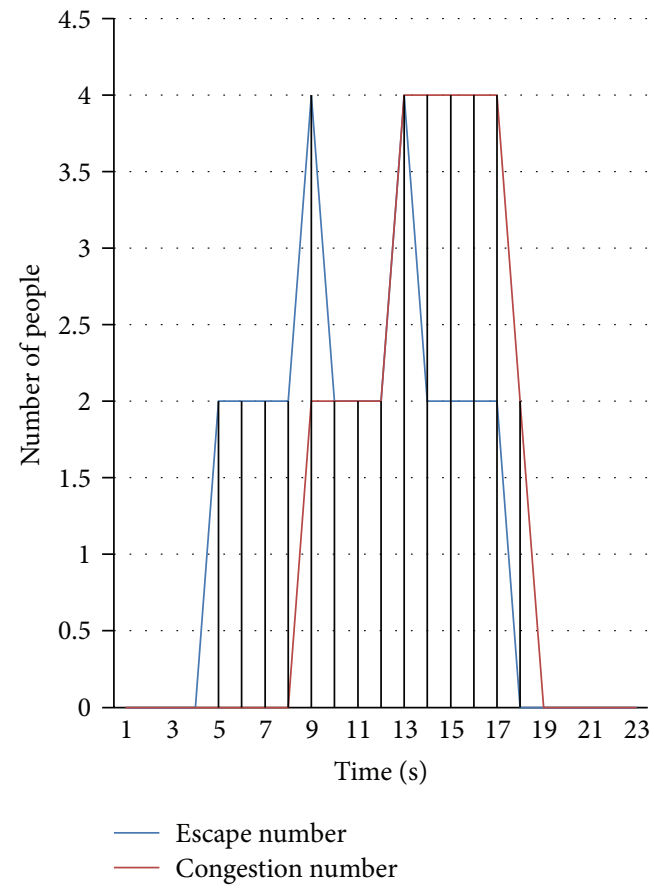

(d) Exit 4

FIgURE 5: Simulation output of each exit.

the agent method in Table 8. We set PIC $=0.5, k=0.1$, $\mathrm{PC}=0.5$, and $r=0.8$ in the snowdrift game theory.

Since snowdrift game theory merely considers a single room, we introduce the shortest route to the global optimization of evacuation planning, in order to compare with our approach.

The performance of the exit selection model obtains a better result than the snowdrift game theory. However, the exit selection model does not consider fire source positions or
MFR. In addition, the agent and snowdrift game theories are microscopic optimizations for evacuation and merely obtain locally optimal solutions, so they cannot arrive at a globally optimal solution from a local optimum in a complex scene. Table 8 shows that our proposed algorithm improves the efficiency of the evacuation. Our method can minimize time compared to other methods. We can conclude that the method guides evacuations to achieve larger outflow and lower probability for congestion at the exit. 


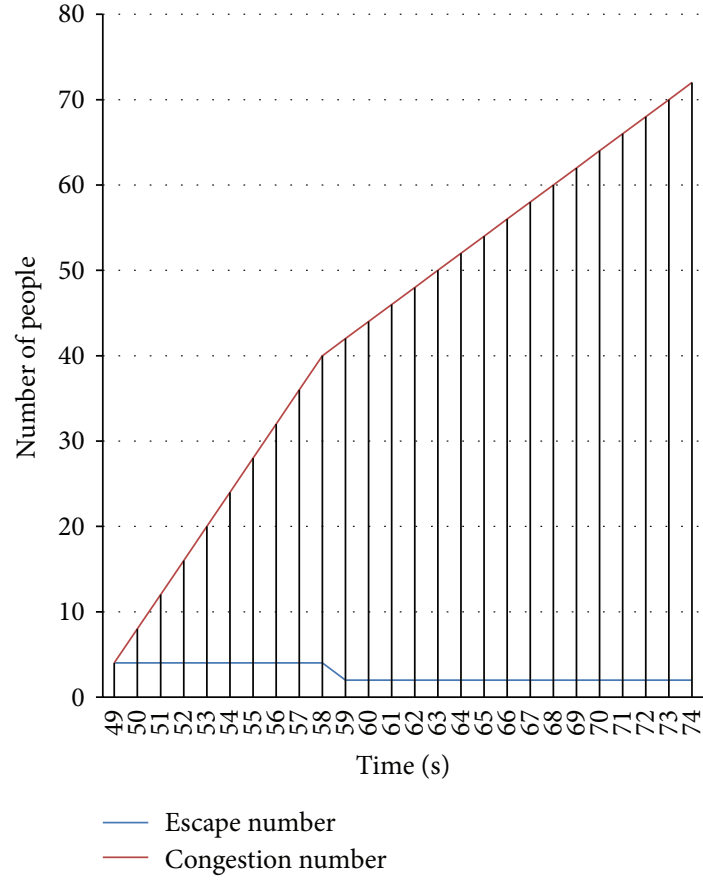

(a) Exit 2

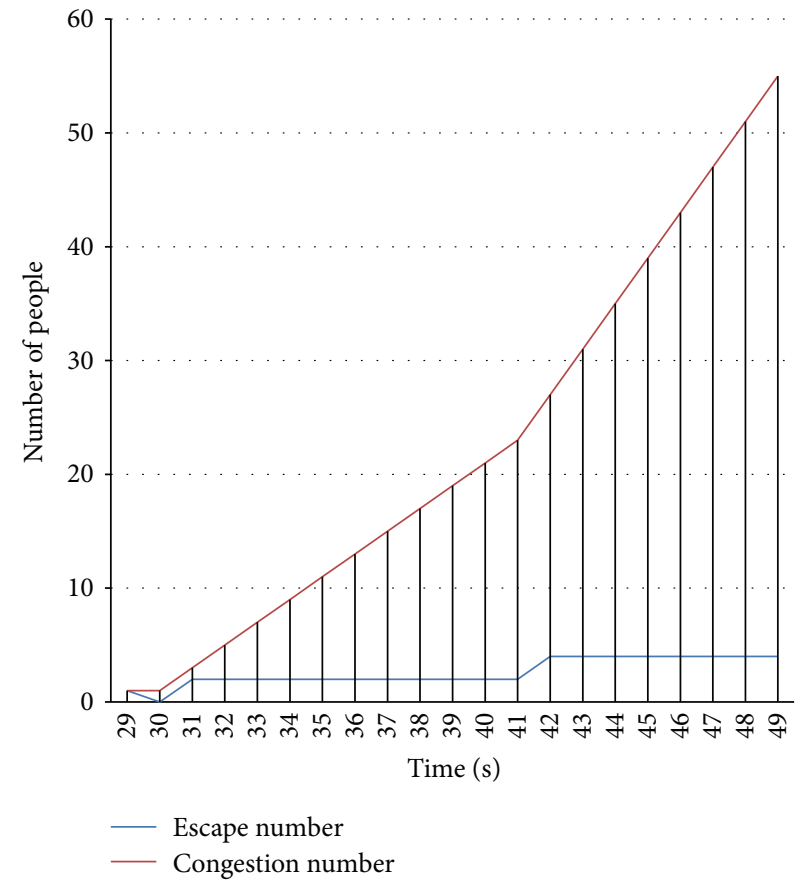

(b) Exit 3 and Exit 4

Figure 6: Expected evacuation numbers of each exit. (a) The pedestrian evacuation distribution at Exit 2. (b) The pedestrian evacuation distribution at Exit 3 and Exit 4.
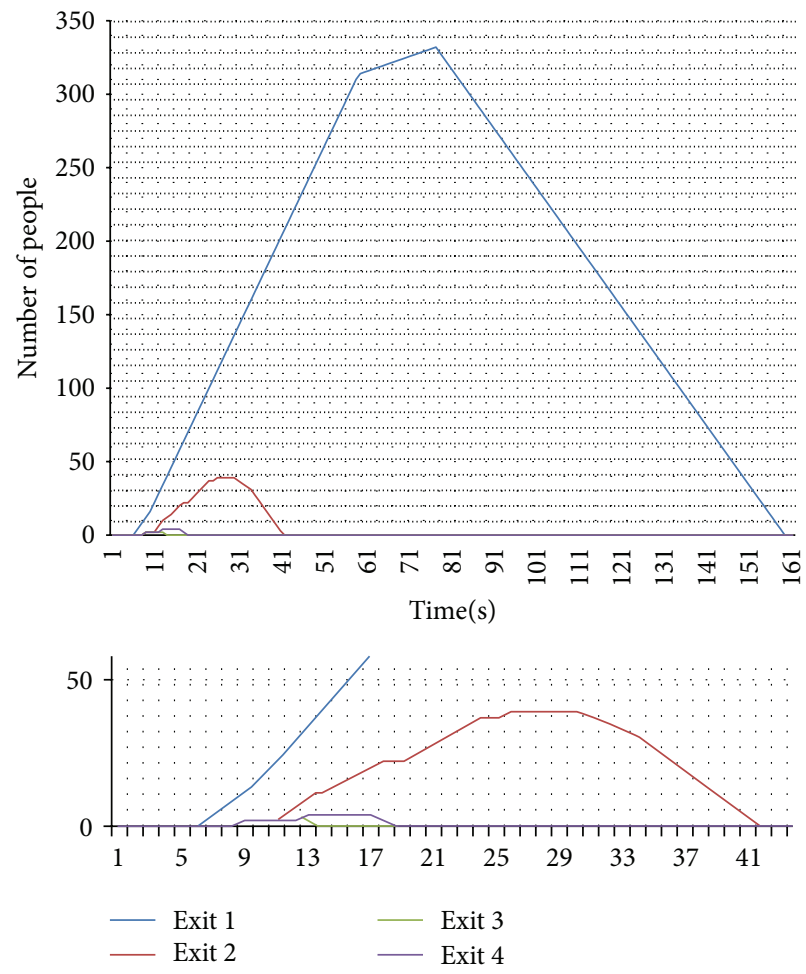

FIGURE 7: The mere consideration of the travel distance from the exit. 


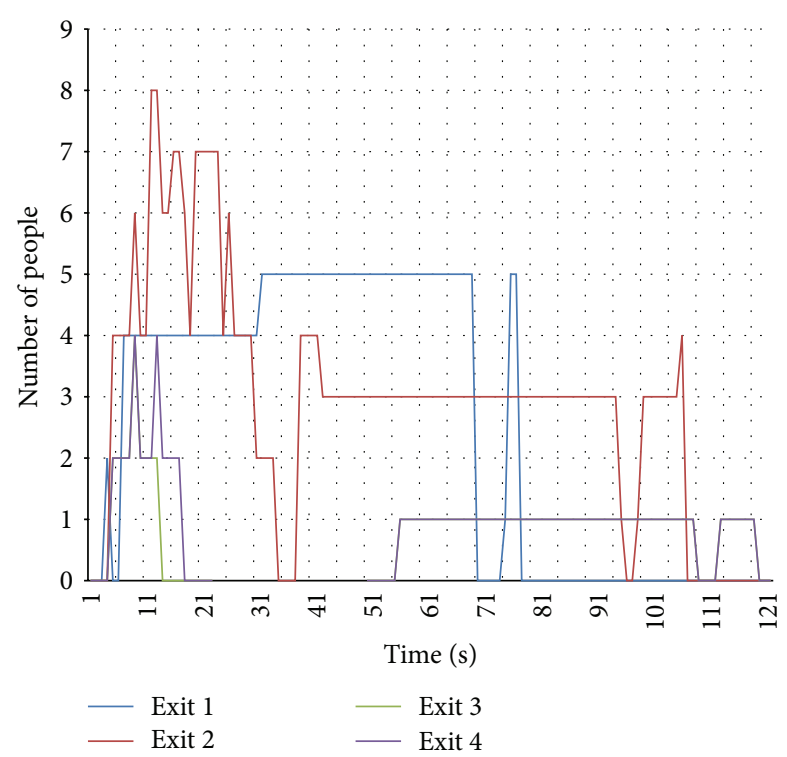

(a) The pedestrian evacuation plan

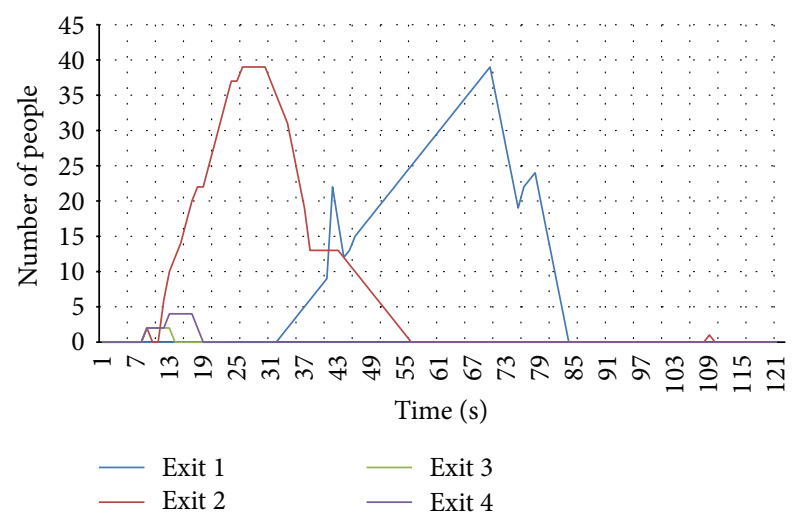

(b) The pedestrian congestion diagram

FIGURE 8: Optimizing the evacuation effect diagram.

TABLE 7: QRA degree list of each exit.

\begin{tabular}{lccccc}
\hline Exit & EC & PC & IC & PTC & QRA \\
\hline 1 & 43.02 & 12.38 & 7.97 & 73.96 & 100.81 \\
2 & 0 & 0 & 7.83 & 36.21 & 44.04 \\
3 & 0 & 0 & 7.96 & 55.13 & 63.09 \\
4 & 0 & 0 & 7.86 & 55.98 & 63.84 \\
\hline
\end{tabular}

EC: export congestion, PC: path congestion, IC: intersection congestion, and PTC: path time-consuming.

TABLE 8: Evacuation characteristics of the contrast table.

\begin{tabular}{lc}
\hline The evacuation method & Time (s) \\
\hline MEEM & $121 \mathrm{~s}$ \\
Agent [5] & 161 \\
Exit selection model [13] & 132 \\
Snowdrift game theory [12] & 156 \\
\hline
\end{tabular}

\section{Conclusion}

Evacuation optimization technology has traditionally faced the problem of achieving effective evacuation planning with minimal evacuation clearance time for individuals. In this paper, we have presented a novel model (MEEM) in which game theory and Monte Carlo methods are combined for evacuation routing optimization in complex scenes. The improved game theory method finds the global minimizer for the evacuation time using maximum entropy theory. This model obtains a global optimum agent distribution with an estimation of the degree of risk of a route to manage the routing selection problem and the congestion conflict problem. Compared to other evacuation models, we employed a method based on an agent whose motion is governed by Newton's equations to simulate the effect of complex building architectures during urgent evacuation. MEEM has been established to examine how the rational evacuation planning of the evacuees affects the evacuation process. Our model considers the exits as participants rather than agents, which can deal with more agents in the game optimization problem. Finally, we develop evacuation plans by calculating payoff functions for convergence to Nash equilibria, which are established based on maximum entropy theory. Thus, MEEM can rationalize the route of the evacuees. Further work will need to examine the effect of familiarity and environmental stimuli as well as accident prevention effects on multiple-exit selection.

\section{Conflict of Interests}

The authors declare that there is no conflict of interests regarding the publication of this paper.

\section{Acknowledgments}

The work described in this paper was funded by National Natural Science Foundation of China (41001302) and Natural Science Research Foundation of Jilin Province of China (20140520071JH and 20120305).

\section{References}

[1] J. Sewall, D. Wilkie, and M. C. Lin, "Interactive hybrid simulation of large-scale traffic," ACM Transactions on Graphics, vol. 30, no. 6, article 135, pp. 1-18, 2011.

[2] Z. Liu, P. Huang, Y. Xu, C. Guo, and J. Li, "The quantitative risk assessment of domino effect caused by heat radiation," in Proceedings of the 5th International Conference on Systems (ICONS '10), pp. 120-124, April 2010. 
[3] X. Pan, C. S. Han, K. Dauber, and K. H. Law, "Human and social behavior in computational modeling and analysis of egress," Automation in Construction, vol. 15, no. 4, pp. 448-461, 2006.

[4] A. I. Parlak, J. H. Lambert, T. M. Guterbock, and J. L. Clements, "Population behavioral scenarios influencing radiological disaster preparedness and planning," Accident Analysis and Prevention, vol. 48, pp. 353-362, 2012.

[5] V. Ha and G. Lykotrafitis, "Agent-based modeling of a multiroom multi-floor building emergency evacuation," Physica A: Statistical Mechanics and Its Applications, vol. 391, no. 8, pp. 2740-2751, 2012.

[6] N. Chooramun, P. J. Lawrence, and E. R. Galea, "An agent based evacuation model utilising hybrid space discretisation," Safety Science, vol. 50, no. 8, pp. 1685-1694, 2012.

[7] M. Manley and Y. S. Kim, "Modeling emergency evacuation of individuals with disabilities (exitus): an agent-based public decision support system," Expert Systems with Applications, vol. 39, no. 9, pp. 8300-8311, 2012.

[8] X. Zheng, T. Zhong, and M. Liu, "Modeling crowd evacuation of a building based on seven methodological approaches," Building and Environment, vol. 44, no. 3, pp. 437-445, 2009.

[9] X. Zheng and Y. Cheng, "Conflict game in evacuation process: a study combining cellular automata model," Physica A: Statistical Mechanics and Its Applications, vol. 390, no. 6, pp. 1042-1050, 2011.

[10] X. Zheng and Y. Cheng, "Modeling cooperative and competitive behaviors in emergency evacuation: a game-theoretical approach," Computers \& Mathematics with Applications, vol. 62, no. 12, pp. 4627-4634, 2011.

[11] J. Tanimoto, A. Hagishima, and Y. Tanaka, "Study of bottleneck effect at an emergency evacuation exit using cellular automata model, mean field approximation analysis, and game theory," Physica A: Statistical Mechanics and Its Applications, vol. 389, no. 24, pp. 5611-5618, 2010.

[12] D.-M. Shi and B.-H. Wang, "Evacuation of pedestrians from a single room by using snowdrift game theories," Physical Review E, vol. 87, no. 2, Article ID 022802, 6 pages, 2013.

[13] S. M. Lo, H. C. Huang, P. Wang, and K. K. Yuen, "A game theory based exit selection model for evacuation," Fire Safety Journal, vol. 41, no. 5, pp. 364-369, 2006.

[14] W. Li, J. Zhu, H. Li et al., "Game theory-based optimal escape route generating method for large-scale crowd evacuation," International Patent, 103279800, 2013.

[15] M. Krstic, P. Frihauf, J. Krieger, and T. Başar, "Nash equilibrium seeking with finitely- and infinitely-many players," in Proceedings of the 8th IFAC Symposium on Nonlinear Control Systems (NOLCOS '10), pp. 1086-1091, Bologna, Italy, September 2010.

[16] S. Hoogendoorn and P. H. L. Bovy, "Simulation of pedestrian flows by optimal control and differential games," Optimal Control Applications and Methods, vol. 24, no. 3, pp. 153-172, 2003.

[17] W. C. Stirling, M. A. Goodrich, and D. J. Packard, "Satisficing equilibria: a non-classical theory of games and decisions," Autonomous Agents and Multi-Agent Systems, vol. 5, no. 3, pp. 305-328, 2002.

[18] X. Yu and Q. Zhang, "Fuzzy Nash equilibrium of fuzzy nperson non-cooperative game," Journal of Systems Engineering and Electronics, vol. 21, no. 1, pp. 47-56, 2010.

[19] D. G. Milledge, S. N. Lane, A. L. Heathwaite, and S. M. Reaney, "A Monte Carlo approach to the inverse problem of diffuse pollution risk in agricultural catchments," Science of the Total Environment, vol. 433, no. 1, pp. 434-449, 2012.
[20] Q. Meng, X. Qu, Y. K. Thong, and W. Y. Heng, "QRA modelbased risk impact analysis of traffic flow in urban road tunnels," Risk Analysis, vol. 31, no. 12, pp. 1872-1882, 2011.

[21] Q. Wang, D. M. Kilgour, and K. W. Hipel, "Fuzzy real options for risky project evaluation using least squares Monte-Carlo simulation," IEEE Systems Journal, vol. 5, no. 3, pp. 385-395, 2011.

[22] M. Marios, "Algorithmic game theory," Theory of Computing Systems, vol. 48, no. 1, pp. 78-86, 2010. 


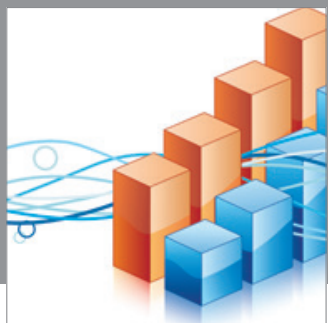

Advances in

Operations Research

mansans

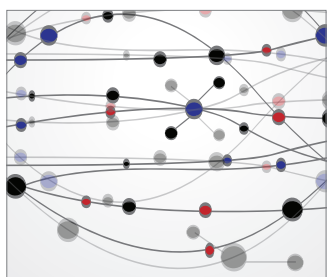

The Scientific World Journal
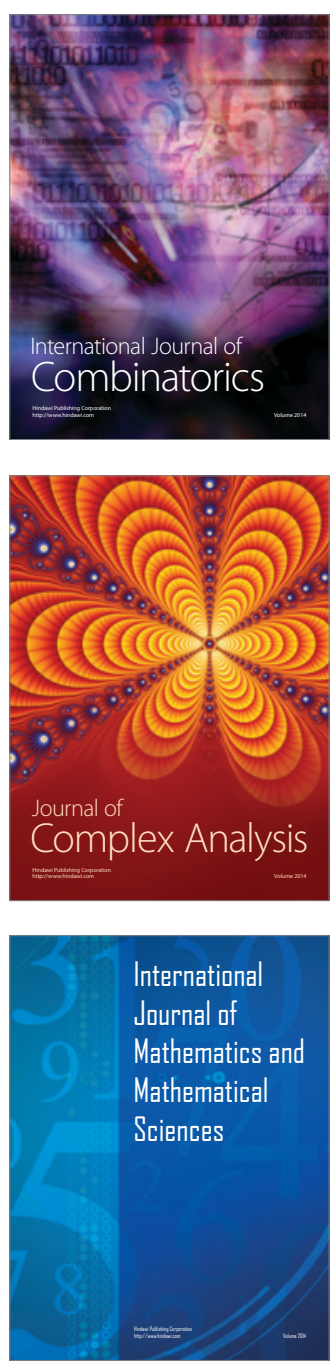
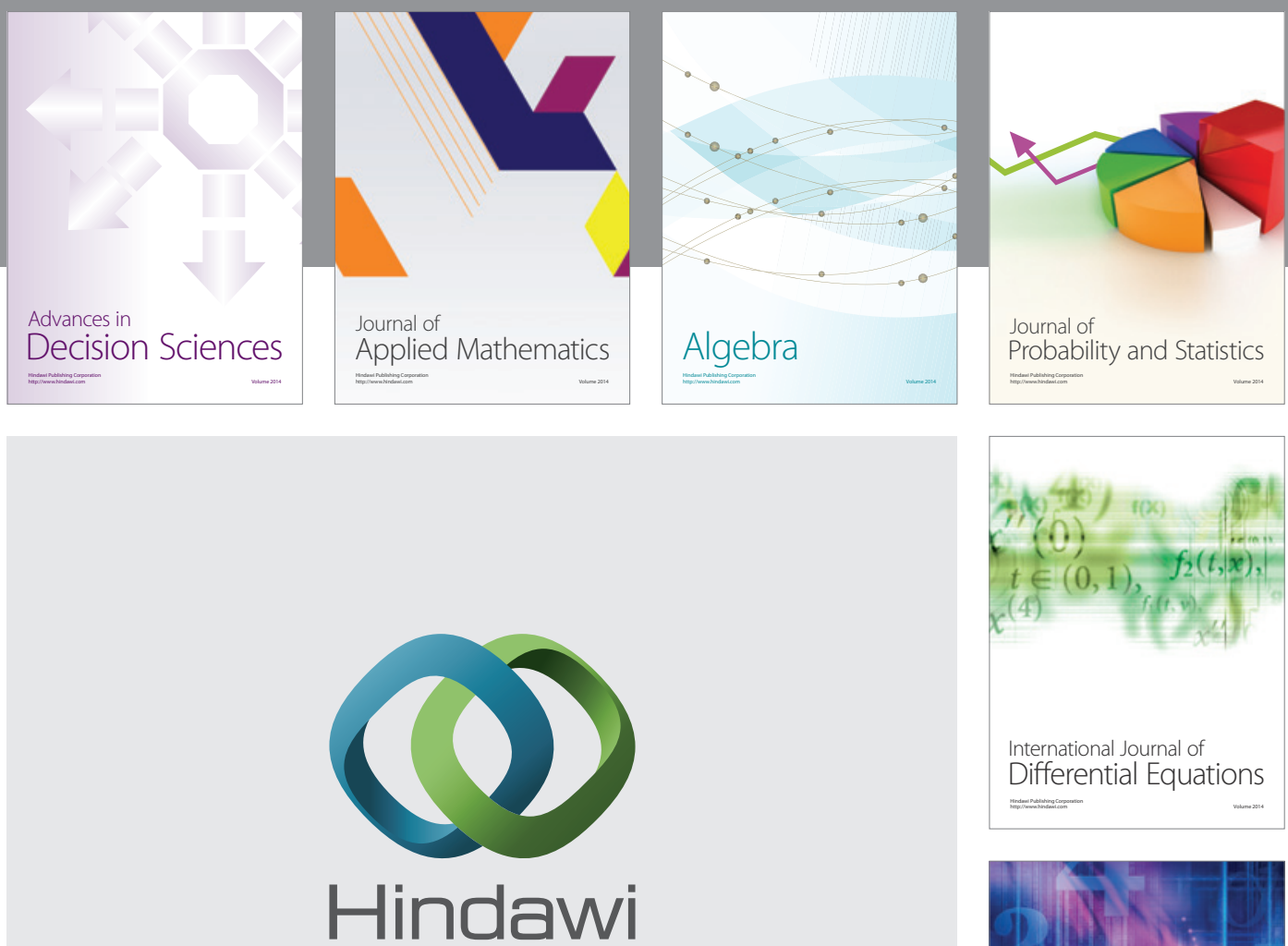

Submit your manuscripts at http://www.hindawi.com
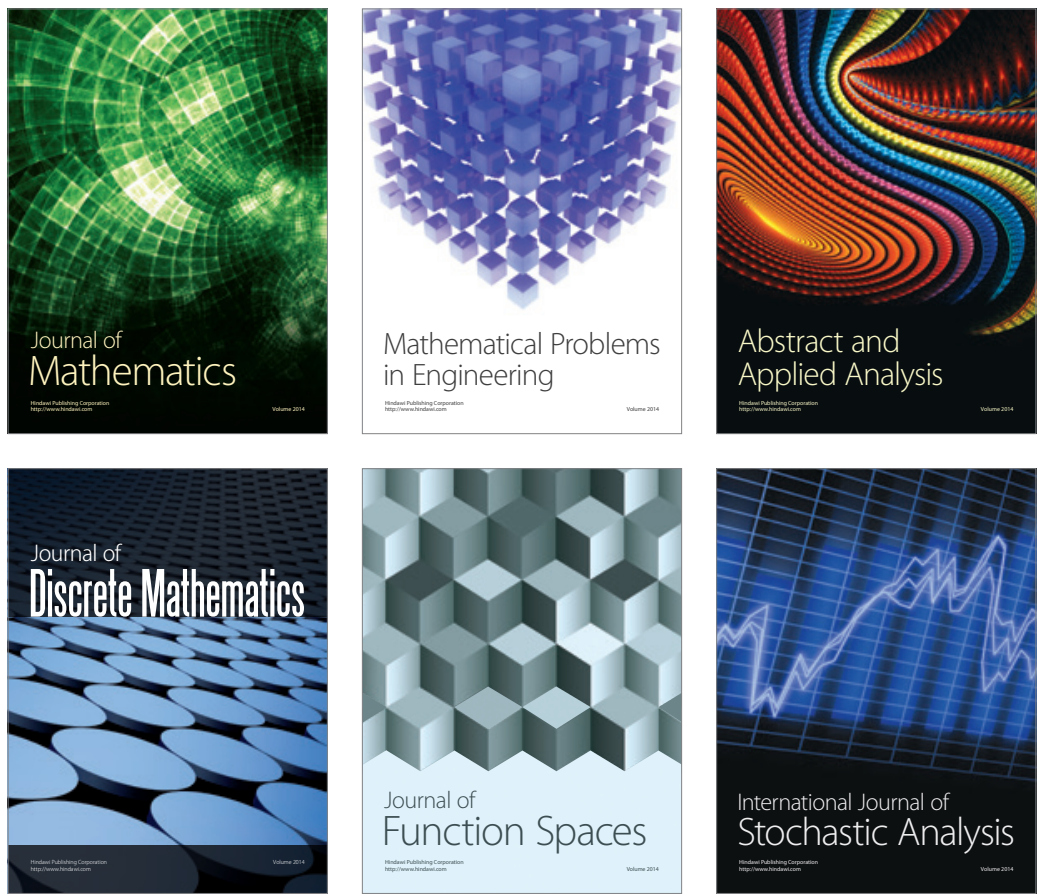

Journal of

Function Spaces

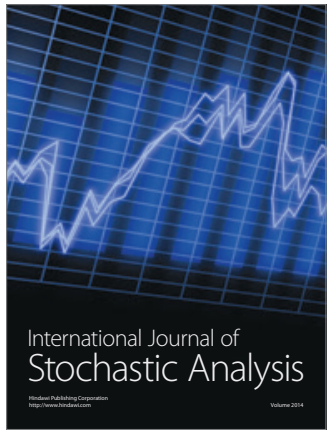

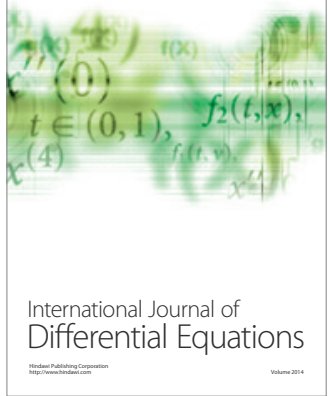
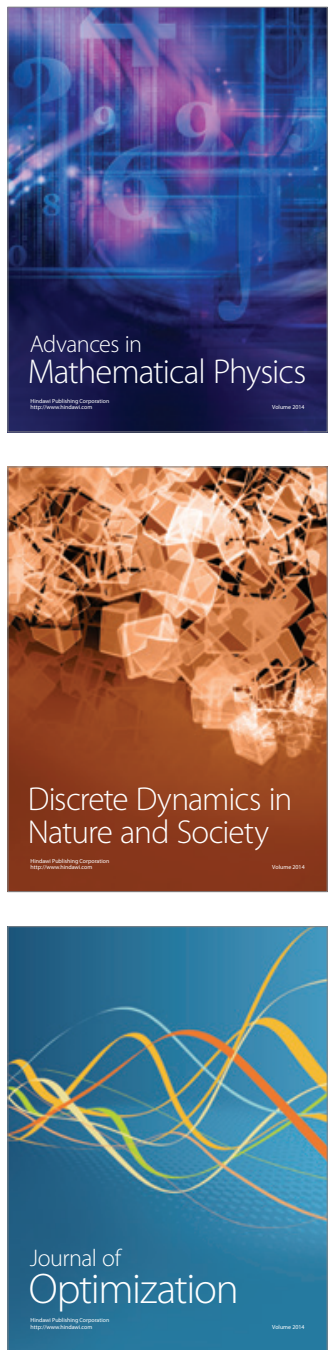